from the soil and from the air and tested their influence on the growth of Pythium. He found that Penicillium expansum was the most markedly antibiotic, and adopting Fleming's procedure, obtained sterilized filtrates: these inhibited the growth of Pythium debaryanum at dilutions of $1: 1,280$. $\mathrm{He}$ did not isolate the antifungal substance, which Anslow, Raistrick and G. Smith (1943) have shown to be patulin. When the fungus was added to garden soil infected with Pythium, seedlings remained healthy instead of damping off.

Here we seem to have a linking up of different lines of research in such a way that we may expect considerable progress. The recognition of the chemical constitution of a substance produced by a common soil fungus which suppresses the growth of a plant pathogen suggests that greater precision may soon be given to many of the older observations on fungal antagonism of various kinds. Incidentally, it may also have some bearing on the question of natural compost versus chemical fertilizers. There is a difficulty in establishing the growth of antagonistic organisms in the soil; this can be done only by modifying the conditions, and an obvious way to do this is to add manure or compost which favour fungal growth. That the matter is not quite so simple as it appears at first sight, however, may be judged from the fact that Barnum in 1924 showed that the filtrate of cultures of Penicillium expansum caused wilting in certain herbaceous plants placed in it.

No more than mention can be made of the production by fungi of fats, ethyl alcohol, lactic acid, vitamins, and enzymes, or of the immense fermentation industries of the Orient.

\section{PLASTICS AND ELECTRICAL TECHNOLOGY}

$P$ LASTICS and electrical technology are close allies on the industrial front, with many points of contact, and in recent years meetings have been arranged at which members of the two professions can exchange views and comments on recent de. velopments of common interest. The latest meeting of this kind, arranged by the Plastics Group of the Society of Chemical Industry, was held at the Institution of Electrical Engineers on April 14, when the topics selected. were the electrical properties of the newer thermoplastics, radio-frequency heating, and 'tracking'.

In opening the discussion, Mr. H. A. Nancarrow pointed out that in the pure hydrocarbon thermoplastics, poly-ethylene, poly-isobutylene and polystyrene, we have arrived at the almost perfect dielectric, in the sense that their power factors are almost vanishingly small, over the entire range of frequency and temperature encountered in electrical practice. In these materials we have the excellent qualities of pure paraffin wax associated with a wide range of mechanical qualities from great flexibility to brittleness. The power factors of the pure materials are, generally speaking, of the order of $0 \cdot 0003$. Much higher values are sometimes quoted, but they are usually due to impurities; either plasticizers added to facilitate extrusion or moulding of the material, or oxidation products, produced when the material has been overheated in air. These materials have made possible many developments in electrical communica- tion at the highest frequencies, where even the smallest power factor, or power loss per cycle, may be very significant. At lower frequencies where higher power factors can be tolerated, thermoplastics like poly-vinyl chloride, which is doing valuable service as a rubber substitute in power cables, and polymethyl methacrylate, well known as 'Perspex', are being widely used. The power factors of these materials are of great scientific interest. They show the characteristic of all polar materials-a high maximum value at a particular frequency or periodic time corresponding with the relaxation time of the molecule. The work of Fuoss and other workers in America on the effect of plasticizers on this molecular relaxation was reviewed, but the discussion showed that though the spade work is progressing well, it has not yet reached the stage where important generalizations are possible.

In opening the discussion on the second topic, Dr. L. Hartshorm remarked that it has recently received a great deal of publicity under such very questionable terms as 'radiotronic' heating and 'heatronic' processes. These newly coined terms seem to be entirely unnecessary. The term 'radio-frequency heating' is satisfactory, but it includes eddy-current heating of metals at radio-frequencies, as well as that of plastics and other insulating materials by the process under discussion, which Dr. Hartshorn suggested should be called 'dielectric heating'. The meeting appeared to be prepared to accept the suggestion. The basic facts have long been known. Any solid or liquid insulating material placed near a conductor which is maintained at a high alternating voltage of high frequency becomes more or less hot. The heat is generated throughout the whole mass of the material, the rate of conversion of electrical energy into heat in any element of volume being proportional to the square of the electric field-strength in that element. The basic experimental facts were forced on the attention of radio engineers in the earliest days of high-power wireless transmitters : insulating supports frequently became so hot as to catch fire, and much research has been needed to discover materials like the pure hydrocarbons discussed by Mr. Nancarrow in which the development of heat is as small as possible, and those which will resist better the action of the heat that is developed. The new ceramic materials are of this class.

The idea of utilizing this generation of heat for industrial purposes seems to have originated in various quarters about fifteen years ago, though it has only quite recently been put into practice on any extensive scale. American patent literature from 1930 onwards describes various schemes for using dielectric heating for sterilizing milk and other foods, killing pests in plant bulbs, drying artificial sponges and tobacco, cementing plywood and safety glass, and leather soles on to shoes, and so on. At first sight it seems curious that the method is still a novelty in industry; no doubt economic factors as well as the technical difficulties have played their part here.

The importance of the process lies in the fact that it makes possible the rapid and uniform heating of thermal non-conductors of any thickness. In a large: American plant stacks of plywood $1 \mathrm{ft}$. thick are said to be heated through a temperature range of $160^{\circ} \mathrm{F}$. in five minutes, this rise of temperature being required for the setting of the urea-formaldehyde glues employed in the making of the plywood. The method has also proved valuable in the plastics 
industry for the heating of moulding materials as a preliminary to moulding. The uniformly heated material flows better into the mould and gives a more uniform moulding than material heated by a contact method, while the rapid heating reduces the time required for the complete moulding process.

An attractive feature of the process is that it makes possible the selective heating of a mixture or composite structure. Thus in fields of a given strength and frequency, the rates of generation of heat in different materials are proportional to the product of their dielectric constant and power factor. The power factor of plate-glass is very small and almost invariable over the whole range of radio frequencies ; that of cellulose acetate rises to a high value at a frequency of the order of $10 \mathrm{Mc}$./s. Thus if alternate layers of plate-glass and cellulose acetate are placed in a high-frequency electric field, the plastic will generate sufficient heat to be brought to the softening point while little heat is generated in the glass. The prospect of facilitating the manufacture of safety glass in this way is one of the most attractive features of dielectric heating.

Among plastics it seems likely that the process will be limited to materials which show an absorption band, that is, a more or less sharp maximum power factor in the radio-frequency range : other materials would require excessive voltages in order to generate sufficient heat. Thus the heat may be considered to originate in oscillations of the polar groups of the materials under the action of the alternating electric field. The question was raised: Is it possible that such heating at particular frequencies may be associated with special chemical activity, and therefore be of special value in processes like the curing of resins ? There appears, however, to be no evidence to suggest that such processes are stimulated by an electric field and selective as to frequency.

The advantages that may be gained by dielectric heating are so great that it may well revolutionize many industrial processes. It is, however, necessary to recognize that it has its limitations. First, as a method of generating heat it is bound to be expensive. In order to get the energy into the form of a high-frequency electric field, it must go through various transformations: low-voltage A.c. to highvoltage A.c. ; then to high-voltage D.C. via rectifiers; then via thermionic valves to the final form. Each transformation requires equipment which may be expensive, and valves being high-resistance generators necessarily involve considerable losses of energy. The result is that dielectric heating cannot compete with ordinary electric heating (conductor heating) or steam heating for processes in which these are effective and not unduly slow. There is also the difficulty of avoiding interference with radio communication. This can, of course, be done by adequate screening, but this adds to the cost and may limit the ease of operation of the process.

A more fundamental limitation arises from the fact that the conditions for uniform heating are not always easily realized, and when uneven heating occurs there may be a risk of damaging the material. There is no maximum temperature which cannot be exceeded, like that of the steam in steam heating. In certain conditions the process is an unstable one. Thus if, as is not uncommon, the dielectric constant and power factor of a material increase with rise of temperature, then the effect of a rise of temperature is to increase the rate of heating, so that the action is cumulative. If this occurs throughout the mass, it will become obvious and the voltage will be reduced either by the operator or automatically as the heat. ing proceeds. But if it is local, it may be undetected since it is the interior of the material that is usually hottest. Obviously then the material to be heated must be carefully controlled as to its uniformity and dielectric properties. Uniform heating will then be obtained provided the electric field is uniform. This again is not always a simple matter. A uniform field can be obtained between two plane parallel electrodes large in comparison with their distance apart, but as the distance apart increases in relation to the size of the plates there is a concentration of field near the surface of the metal. Occasionally this may be useful in counteracting the cooling effect of the electrodes; but in other cases it is necessary to have an air gap between the material and the electrodes to avoid excessive heating of the surface layers.

There can be little doubt that the process wlll lead to important developments in plastics technology. The ability to heat large thicknesses uniformly makes possible the manufacture of laminated material such as is used for gear wheels in thicknesses previously unknown, and should make possible the production of thicker mouldings. The rapid jointing of materials like leather and fabrics with thermoplastic cements should be possible for sheets of any thickness, while the welding of thermoplastics of any thickness also becomes a possibility. The search for plastics of low power-factor which has had such striking successes in recent years may now be followed by one for materials of very high power-factor in some particular frequency range specially suited to dielectric heating.

The last subject discussed was the breakdown of insulating materials by the process technically known as 'tracking'. This is a localized, but progressive, charring, which, starting at one point, spreads more or less rapidly as a tree-like growth right across the surface of the insulator, finally short-circuiting it and carrying so much current that the insulator is destroyed by the heat generated. In opening the topic, Mr. E. Rushton explained that it is essentially a surface phenomenon, which only takes place on insulators which have been in service for some time, and only occurs in a humid atmosphere. That it is a surface phenomenon is shown by the fact that it can be to some extent prevented by coating the surface of the insulator with special varnishes, but there are the most diverse views as to the nature of the process, and correspondingly diverse opinions about suitable tests for the grading of materials for their tendency to fail by tracking.

It is generally agreed that the phenolic resins are on the whole more liable to fail in this way than urea resins, and it was stated that melamine resins are even better than urea ones in this respect; but it was suggested that the difference is more likely to be one of macro-structure than molecular constitution. The final stage of the process is clearly dependent on a heterogeneous surface structure; but how far this is characteristic of the resin itself and how far dependent on the chemical effect of surface moisture under the sustained voltage gradients in the surface is still a matter of conjecture. Stäger has described the process as analogous to the fatigue corrosion of metals, and dependent on the salt content of the materials, which gives rise to mobile ions in the films of water on the surface in a humid atmosphere. These ions moving under the action of the surface 
voltage gradients lead to local deposits of salt and local concentrations of field strength, and therefore to local heating, which starts the formation of a conducting track of carbonized material. Once it has started it will inevitably travel down the voltage gradient, for it will behave like a pointed conductor at the tip of which there will always be a strong concentration of field strength and therefore local heating when the surface conductivity is appreciable.

Another view put forward was that tracking is always started by sparking over the surface, the steep voltage gradient required to produce a spark arising where the surface of the insulator becomes partially bridged by patches of relatively high conductivity, leaving narrow gaps of low conductivity to support an undue share of the total voltage. The conducting patches may arise from dirt or irregular wetting as the humidity rises.

Tracking can certainly be deliberately initiated by applying a high voltage and so causing sparks to pass over an insulating surface between electrodes with sharp edges, or alternatively by spraying the insulating surface with salt solution and so causing irrogular wetting while a comparatively low voltage is applied; and these methods are used as rapid tests of the tendency of materials to break down by tracking. Their reliability as an index of performance in practice is not, however, universally conceded. A quick test would be of great practical importance in the development of 'anti-tracking' plastics, but a controlled procedure which will reproduce accurately the essential conditions will only be possible when the phenomenon is better understood.

Dr. Haefely somewhat ruefully summarized the immediate practical results of the meeting by saying that, as a manufacturer, he would, under present conditions, have to continue to make the best materials he could, to wait for apparatus for dielectric heating, and to design his electrodes so as to discourage the initiation of tracking.

\section{HARTSHORN.}

\section{SCIENCE AND ART AT THE ROYAL ACADEMY, 1944}

\author{
By Dr. A. T. HOPWOOD \\ British Museum (Natural History)
}

$\mathrm{I}^{\mathrm{N}}$

$\mathrm{N} 1942$ I attempted ${ }^{1}$ to explain what I understand art to be. Since some of my gossips later took me to task because neither beauty nor truth were mentioned, it may be well to point out that beauty is not essential to a work of art, and that truth is an ambiguous word often used to mean accurate representation.

Consider, for example, the numerous flower pieces in the present Royal Academy Exhibition; these range from highly finished coloured drawings to mere blobs of paint placed in such a way as to give the impression of flowers. A botanist or a gardener will probably prefer the former, since the flowers are easy to identify, and that makes for self-confidence in the observer, who feels that he understands what the painter is trying to do, though whether that feeling is justified is perhaps another matter. An artist, on the other hand, may well prefer the blobs; he thinks more in terms of harmonies, contrasts and design, and notices how the whole is enlivened by discreet touches of discordant colour, and how by proper emphasis the whole thing is made to hang together. In the popular sense of the word, only the first type of painting is true, whereas in fact both are true. Provided that this ambiguity is recog. nized, one is justified in the claim that truth is an essential component of art; but it was to avoid any misunderstanding that I spoke of intellectual honesty instead.

With so high 'a standard in the exhibits, the critic this year is fortunate in his lot. There are numerous items to which one would like to direct attention, but most of them are excluded by the title of this notice. Of those which remain, pride of place must be given to "Night Raid, 1941" by Richard Eurich (No. 710). Although it was painted "for the Nation's War Records", this work has about it a quality which lifts it above the competent journalism of the average war reporter. The canvas is full of incident, it tells not one story but many; the contrast between the hot fiery glow and the cold glare of the searchlights is admirably handled, but the hard white mount is not so well inspired. More than all these is a quality of innocence, as though the artist had approached his subject with the unspoilt mind of a child and recorded the night's events with a simple directness which more sophisticated minds would label primitive. "Antwerp" (No. 17) by the same artist is in a different mood ; it was painted before the War began and is one of the Chantrey Bequest purchases; good picture though it is, "Night Raid" is better.

In so far as it is possible for one artist to steal the show, that has been done by A. R. Thomson. Look at his two pictures, "Apple Trees" (No. 97) and "Miss Joan Chapman" (No. 102), which hang a few feet apart in Gallery II. The trees really grow out of the grass ; the spraying and the pruning may have been neglected, but the whole picture is redolent of the average farmer's orchard. In complete contrast is the relaxed body of the dancer, whose weight is taken down well and truly to the right foot. Then go round the corner and read the character of the chairman of the London County Council in his portrait (No. 224), and, finally, look at the young airmen having a happy night in the mess before "Going to be Decorated" (No. 754). Such versatility does not appear very often.

As for the purely scientific aspects of the Exhibition, the anatomist should enjoy the portrait of Miss Chapman just mentioned, and medical men Nos. 292 and 746-the titles are too long to quote. The portrait of Sir Henry Dale by Francis Dodd (No. 140) is not convincing, and that of Sir John Russell by Francis Hodge (No. 335) is irritating because the direction of the sitter's gaze, towards the bottom left corner of the frame, continually leads one's eyes away from the centre of the picture and distracts the attention. Here, too, may be mentioned Francis Dodd's charcoal drawing of Sir Charles Sherrington (No. 977) and Alfred Hardiman's bronze, "Study of a Scientist" (No. 1177).

Zoological subjects are few ; most of them are horses which, apart from those by A. J. Munnings, P.R.A., are not particularly good. The best is the group to the right of the centre in Munnings' "Winter Meeting" (No. 17). Not only does the necessary foreshortening pose a difficult problem in perspective, but also the design made by the animal's feet is well-conceived; moreover, the horse and its rider are full of life and motion. One of the most curious 